\title{
Deposition of Silver Nanoparticles on Silicone hydrogels by Using Laser-Assisted Process
}

\author{
Vishnuvardhana Wuppaladhodi' ${ }^{1}$, Jin Zhang $^{1}$ \\ ${ }^{1}$ Department of Chemical and Biochemical Engineering, University of Western Ontario, \\ London, Ontario, N6A3K7, Canada \\ vwuppala@uwo.ca; jzhang@eng.uwo.ca
}

\begin{abstract}
Silicone hydrogels have been extensively used in biomedical applications. However, protein adsorption and bacterial contamination are the major drawbacks of silicone hydrogel due to their hydrophobic property. Matrix assisted pulsed laser evaporation (MAPLE) has shown the capability for depositing soft marerials like organic and biomolecules. In this paper, we use MAPLE processes to deposit silver nanoparticles (Ag NPs) modified with polyvinylpyrrolidone (PVP) on silicone. The average particle size of Ag nanoparticles deposited by MAPLE was estimated at $15 \pm 3 \mathrm{~nm}$. The mechanical properties of the nanocomposite coated silicone hydrogel were investigated and the value of Young's modulus was found to be $0.62 \mathrm{MPa}$. Moreover the protein adsorption on the nanocomposite coated silicone hydrogel formed by two step was found to be $14.109 \mu \mathrm{g} / \mathrm{cm}^{2}$.
\end{abstract}

Keywords: Silicone hydrogel, protein fouling, matrix assisted pulsed laser evaporation (MAPLE), silver nanoparticles

\section{Introduction}

Hydrogels have been one of the best choices for biomedical applications due to their unique biocompatibility, flexible methods of synthesis, high water content and desirable physical characteristics [1-2]. Hydrogel is a network of interconnected chains of polymers that are hydrophilic in nature. Hydrogels are capable of containing over $90 \%$ water in between their polymeric networks. Hydrogels have been extensively used in scaffolds in tissue engineering [3], drug delivery devices [4], contact lenses [5], etc.

Silicone hydrogels have been extensively used in tissue engineering, drug delivery systems, contact lenses, etc. due to their good biocompatibility and excellent oxygen permeability. Silicone hydrogel has a different oxygen transport mechanism compared to other hydrogels due to the siloxane groups present in it, which can carry a large amount of oxygen. Silicone hydrogel transports oxygen through the siloxane-phase rather than through the water phase [6] which results in higher oxygen transmissibility than the other hydrogels [7]. High oxygen permeability is one of the desirable characteristics of silicone hydrogel. However, their hydrophobic nature makes them difficult to wet.

Adsorption of protein onto the surface of biomaterials is undesirable as it reduces its efficiency and causes harmful side effects in case of biomedical devices. Protein adherence to the surface of silicone hydrogel involves van der waal forces, electrostatic interactions, and hydrogen bonding. Based on the interaction between water (present in the surface) and proteins there are two types of surfaces; one is hydrophilic surface and the other is hydrophobic surface. When the surface of silicone hydrogel is hydrophobic, the protein molecules tend to rearrange the structure to reach a lower Gibbs free energy [8-9]. The amino acid group of protein which is hydrophobic in nature, will interact with the hydrophobic surface of hydrogels, which will result in denaturing of the protein structure [10]. The denatured protein on hydrophobic surface is irreversible. These denatured proteins may also interact with other proteins, which may cause protein aggregation and cause adverse clinical events [11]. However, hydrophilic surfaces will not denature the protein structure. In fact, hydrophilic surface modification will be an efficient way to prevent irreversible protein adsorption onto the surface of biomaterials. It is also noted that the protein adsorption normally is the first step for the formation of the biofilm which composes of bacteria leading to severe implant-related infection and inflammation [12].

There are two methods to prevent protein adsorption on biomaterials. One is called "attack", and the other is "defense". The "attack" method is to coat a protein degrading film onto the protein adsorbed surface to degrade and remove the adsorbed protein. The "defense" method is to create a protein resistant surface which prevents the protein adsorption in the first place [12]. Polyvinylpyrrolidone (PVP), polyethylene glycol (PEG) based polymers, carbohydrates etc. are few commonly used polymers to modify the surface of biomaterials with a protein resistant surface. 
Polyvinylpyrrolidone (PVP) is an important water soluble synthetic polymers, which has many ideal properties including low toxicity, chemical stability, and good biocompatibility [13], and has been extensively used in daily chemical industry, food, biomedical field, etc. [14] Therefore, PVP is one of the commonly used polymers to improve the hydrophilicity and antifouling properties of the hydrophobic biomaterials.

Similarly, we have two approaches to inhibit the bacterial growth on the surface of the biomaterials; "attack" and "defend". The "attack" approach is to coat an antimicrobial film such as antibiotics, short peptides, cationic polymers, etc. onto the bacteria colonized surface to kill bacteria. The "defend" approach is to create a non-fouling coating, such as PVP, PEG based polymers, inorganic nanoparticles, etc. to resist bacterial adhesion.

Silver-based materials have been studied over the past century [15], due to their extraordinary physico-chemical properties including high electrical and thermal conductivity, chemical stability, surface plasmon resonance, antimicrobial property, surface-enhanced Raman scattering, and catalytic activity [16]. In the field of antibacterial property, silver nanoparticles (Ag NPs), silver metal and silver ions were extensively used for ages [17]. Therefore, surface modification of silicone hydrogel with silver based nanoparticles and polymers such as PVP (more hydrophilic material) has shown improved hydrophilicity.

Compared to chemical coating process, physical methods could avoid the damage of silicone hydrogel caused by chemical agents [18]. Matrix-Assisted Pulsed Laser Evaporation (MAPLE) is a thin film deposition technique derived from Pulsed Laser Deposition (PLD) [19] which helps in deposition of soft materials (polymers, biological materials) without damaging its structure. PLD is one of the most successful ways to deposit high quality thin films, but it is not effective in case of depositing delicate materials. This is because the laser beam is used to heat the target directly (laser ablation) which can damage the structure and properties of the coating material. In the case of MAPLE, the target is a frozen matrix consisting of a volatile solvent, e.g., water, ethylene glycol, chloroform, isopropanol etc., and a low concentration of the film/coating materials [20]. The laser pulse initiates a photo-thermal process, sublimating the frozen solvent, and releasing the coating material into the vacuum chamber. The momentum, resulting from this process, carries the solvent and the coating material to the substrate. As the solvent has a high vapor pressure at room temperature, it will be removed by a mechanical pump in the MAPLE system. The coating material will then adhere to the substrate to form a thin film. The advantage of the MAPLE process is that the laser interaction occurs mostly with the solvent molecules, thus the coating material will not undergo thermal decomposition.

\section{Experimental}

\subsection{Synthesis of Silicone hydrogel}

Photo-polymerization reaction was carried out in this research work to synthesize silicone hydrogel [33]. Briefly, 3methacryloxypropy-tris(trimethylsiloxy)silane (TRIS), bis-alpha, omega-(methacryloxypropyl)polydimethylsiloxane (PDMS) and N,N-Dimethylacrylamide (DMA) were mixed in a calculated volume ratio. Then $15 \mu \mathrm{L}$ of Ethylene glycol dimethacrylate (EGDMA) and $0.18 \mathrm{~mL}$ of 1-Vinyl-2-pyrrolidinone (NVP) were added to the mixture along with $0.3 \mathrm{~mL}$ of ethanol. Nitrogen gas was purged for 15 minutes to remove the dissolved oxygen. Following that, photo initiator Diphenyl(2,4,6-trimethylbenzoyl) phosphine oxide was added to the mixture and stirred for 5 minutes. Then the mixture is poured into the mold and kept in the UV environment for 30 minutes to form complete cross linking. Then Ethanol-water solution (20\%) was used to wash the hydrogel after the polymerization reaction.

\subsection{Coating process by using MAPLE technique.}

As shown in the Fig 1(a) the nanocomposites were prepared by a photo-reduction process which requires exposure to UV environment. These nanocomposites were then suspended in the target holder of the MAPLE device and frozen using liquid nitrogen for thin film deposition as shown in the Fig 1(b). This is the most commonly used method for thin film coating and it has been reported in previous research work. 
(a)

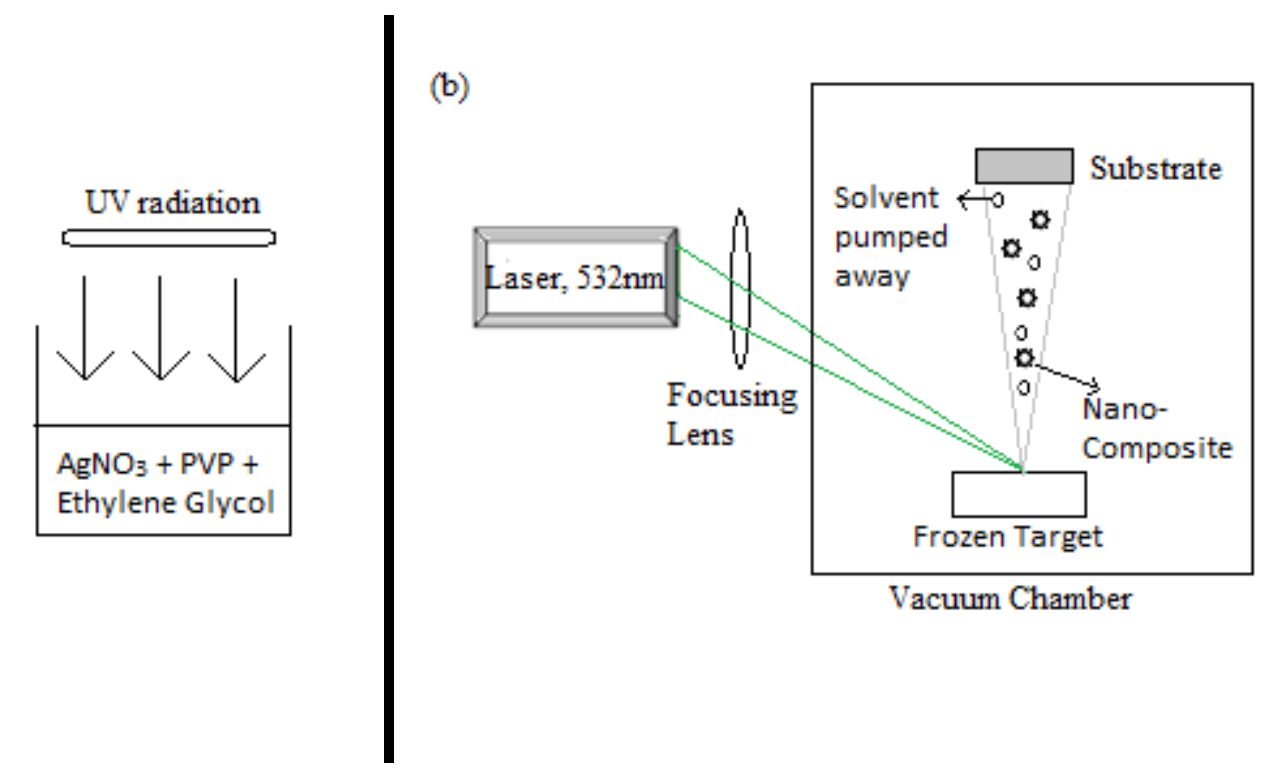

Fig. 1. Two-step process (a) photo-reduction of target solution $\left(\mathrm{AgNO}_{3}, \mathrm{PVP}\right.$ and Ethylene Glycol) by UV radiation (b) deposition of nanocomposites in the vacuum chamber

Nanocomposites which were formed by photo-reduction of silver nitrate with PVP in ethylene glycol were dispersed in the target holder of MAPLE device. The solution was frozen using liquid nitrogen. Nd:YAG laser was used in the MAPLE with a wavelength $(\lambda)$ of $532 \mathrm{~nm}$. The deposition was carried out for 30, 60, 90 and 120 minutes respectively, at a background pressure of $1 \times 10^{-5}$ Torr with a substrate-target distance of $4.5 \mathrm{~cm}$.

\subsection{Measurement of Protein adsorption}

Micro BCA method was used to measure the protein adsorption property of silicone hydrogel. $1 \mathrm{~cm} \times 1 \mathrm{~cm}$ silicone hydrogel samples (bare silicone and Ag-PVP coated silicone) were cut and immersed in Phosphate Buffer Solution (PBS) for 24 hours. Bovine albumin serum-Phosphate Buffer Solution (BSA-PBS) $0.5 \mathrm{mg} / \mathrm{mL}$ and Sodium Dodecyl SulfatePhosphate Buffer Solution (SDS-PBS) 1wt \% solution were prepared. The samples were soaked in $0.5 \mathrm{mg} / \mathrm{mL}$ BSA-PBS solution at $37^{\circ} \mathrm{C}$ for 3 hours. Then these samples were rinsed 3 times in PBS to remove non adsorbed protein on the surface of the hydrogel. Then the samples were immersed in 1wt\% SDS-PBS solution and sonicated for 20 minutes to completely detach adsorbed BSA from the surface of the silicone hydrogel. Finally, the BSA protein assay kit was used to determine the protein concentration in SDS-PBS solution with a UV-Vis plate reader at $562 \mathrm{~nm}$.

\subsection{Mechanical Test:}

$1 \mathrm{~cm} \times 1 \mathrm{~cm}$ samples of silicone hydrogel were cut and mounted on BioTester 5000 test system (CellScale Biomaterials Testing). The samples were stretched uniaxially with a pre-loading of $10 \mathrm{mN}-30 \mathrm{mN}$ or $10 \%-30 \%$ of stretching applied. The images of the deformation of the sample hydrogel were captured using a 1280x960 pixel charge coupled device CCDcamera. The stress and strain were calculated from the data and the Stress-Strain curves of different samples were plotted to determine their Young's modulus (E). The slope of the Stress-strain is the Young's modulus (E) of the sample. The equation to determine the Young's modulus is given below.

$$
\mathrm{E}=\frac{\text { Stress }}{\text { Strain }}=\frac{\sigma}{\varepsilon}=\frac{F / A}{\delta \mathrm{L} / \mathrm{Lo}}
$$

Where $E$ is in Pascal $(\mathrm{Pa}), F$ the force applied in Newton $(\mathrm{N}), A$ the area perpendicular to the force vector $\left(\mathrm{m}^{2}\right), \delta L$ the displacement of the materials $(\mathrm{m})$, and $L_{o}$ the original length of the materials $(\mathrm{m})$. 
For measuring the toughness of the silicone hydrogel sample, maximum stretching is required. Based on the thickness of the silicone hydrogel, $30 \%$ of stretching or more is applied on the hydrogel. It is then calculated from the area under the stress-strain curve. The formula or toughness is given below.

$$
\text { Toughness }=\int \sigma \mathrm{d} \varepsilon
$$

\section{Results and Discussion}

Single-step process is a time saving process and moreover prevents the contamination of target as the nanocomposites are synthesized inside the vacuum chamber. In the two-step process, the synthesis of Ag-PVP nanoparticles requires 24 hours of UV irradiation for the photo-reduction reaction to complete. Once the nanoparticles were synthesized, the solution was suspended in the MAPLE target holder for deposition by laser ablation. Copper grids ( 200 meshes) were placed on the substrate to characterize the nanoparticles. The TEM micrographs in the Fig. 2 show the particle size Ag NPs formed by UV-reduction and deposited in MAPLE for different deposition time.

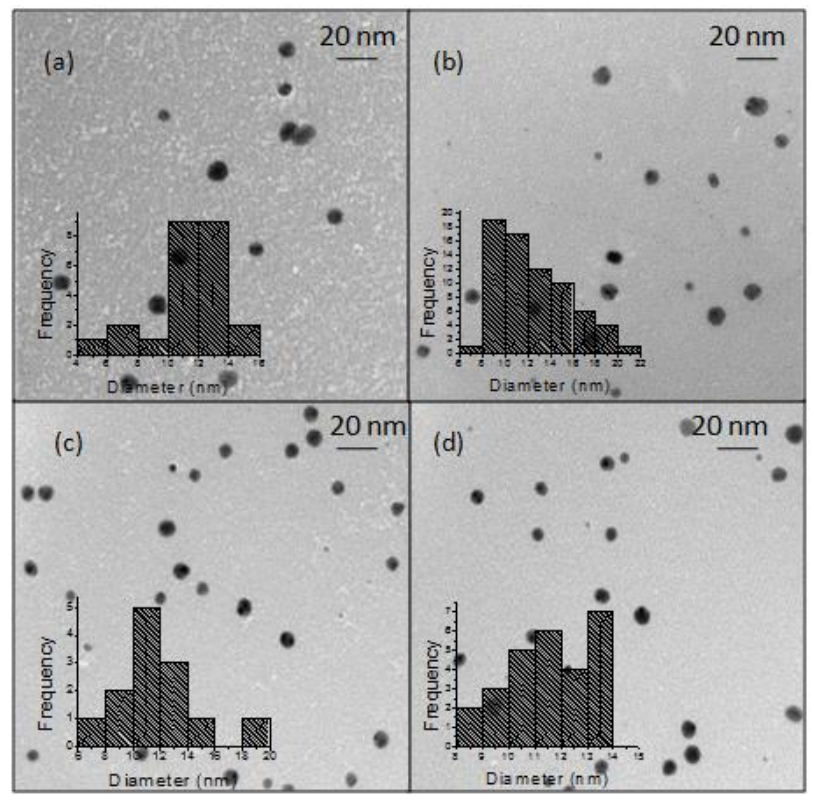

Fig. 2. TEM image of Ag-PVP nanoparticles prepared under UV-radiation and deposited in MAPLE for (a) 30-min (b) 60-min (c) 90-min (d) 120-min

Fig. 3 shows the UV-Vis spectrum of Ag-PVP deposited on silicone hydrogel by both two-step and single-step process as well as bare silicone hydrogel. The adsorption peak which is the surface plasmon resonance (SPR) band was affected by the shape and dielectric environment of nanoparticles. Previous research work states that the Ag NPs will be spherical in shape if the SPR band is around $400 \mathrm{~nm}$ [36]. Therefore, the shape of Ag-PVP NPs should be spherical as depicted from 
the result of TEM micrographs. UV-Vis spectrum of bare silicone which does not show any adsorption peak around 400 $\mathrm{nm}$. The result confirms the presence of Ag-PVP nanocomposite thin film on silicone hydrogel.

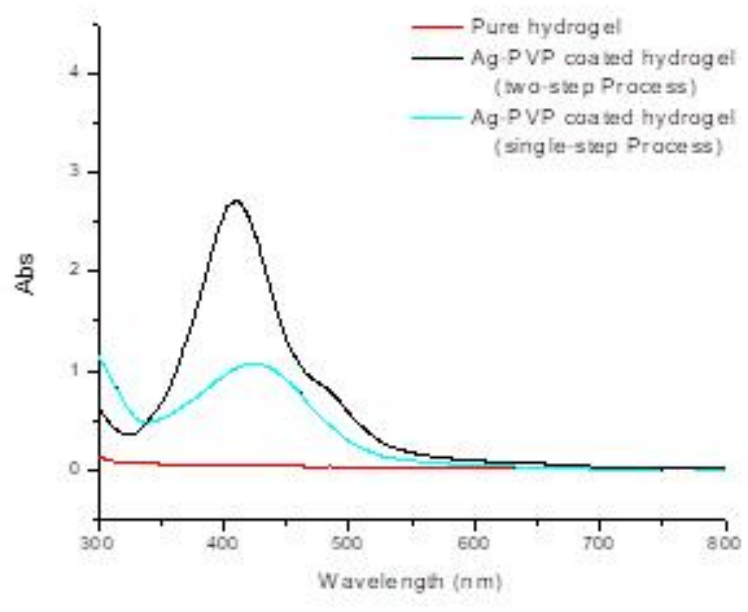

Fig. 3. UV-Visible spectroscopy image of Ag-PVP Nanoparticles deposited on silicone hydrogel by two-step and single-step process as well as bare silicone hydrogel.

BSA protein adsorption of bare silicone hydrogel and Ag- PVP coated silicone hydrogel are $16.095 \pm 0.751 \mu \mathrm{g} / \mathrm{cm}^{2}$, $14.109 \pm 0.683 \mu \mathrm{g} / \mathrm{cm}^{2}$, respectively. It can be inferred that Ag-PVP can reduce the adsorption of non-specific protein due to the presence of polymer (PVP). PVP provides a more hydrophilic surface than bare silicone due to the presence of $\mathrm{C}=\mathrm{O}$ in the structure. When the BSA interacts with hydrophobic surface, it will denature the structure of the protein in order to reach lower Gibbs free energy. But when BSA interacts with hydrophilic surfaces, it will easily get adsorbed onto the surface without changing the structure of the protein, so it is easy to remove the protein from its surface.

The values of the Young's modulus (E) were obtained from the slope of Stress-Strain curve. The value of Young's Modulus of bare silicone hydrogel is $0.57452 \pm 0.1262 \mathrm{MPa}$, Ag-PVP coated silicone hydrogel prepared by two step method is $0.61864 \pm 0.1416 \mathrm{MPa}$. The values of toughness obtained from the area under the Stress-Strain curve is $15.137 \pm$ $0.412 \mathrm{MJ} / \mathrm{m}^{3}$ for bare silicone, $21.542 \pm 0.208 \mathrm{MJ} / \mathrm{m}^{3}$ for Ag-PVP coated silicone hydrogel prepared by the two-step MAPLE.

\section{Conclusions}

In this paper, we have successfully demonstrated the MAPLE process in depositing a nanocomposite on silicone hydrogel. The Ag-PVP nanocomposite film and the size of nanoparticles have been measured by TEM, EDAX-mapping and UV-visible spectroscopy. It was found that as the deposition time increases, the size of nanoparticles formed decreases, but that did not have much effect after exposing it to laser more than an hour but the size distribution is quite even as the deposition time increases and the average size of Nanoparticles formed by single step process was found to be smaller than that formed by two step process. The adsorption peak which is the surface plasmon resonance (SPR) band in the UV-Vis spectrum shows that the silver nanoparticles are spherical in shape. The EDAX mapping and EDAX spectrum shows the elements present in the bare and Ag-PVP deposited silicone hydrogel which confirms the deposition of silver NPs. The BSA protein adsorption test shows that the amount of protein adsorbed after Ag-PVP coated silicone hydrogel was lower than that of protein adsorbed on bare silicone hydrogel. In addition, the Ag-PVP coated silicone hydrogel prepared by MAPLE process $\left(14.109 \mu \mathrm{g} / \mathrm{cm}^{2}\right)$ shows good protein resistant property which could be a method to avoid the formation of biofilm. 


\section{Acknowledgements}

We would like to acknowledge the Natural Science and Engineering Research Council of Canada (NSERC) and Western Engineering Graduate Scholarship.

\section{References}

[1] B. V. Slaughter, S. S. Khurshid, O.Z. Fisher, A. Khademhosseini, and N. A. Peppas, "Hydrogels in regenerative medicine", Adv Mater 21, 3307-3329, 2009.

[2] N. A. Peppas NA, Hilt JZ, Khademhosseini A, Langer R, "Hydrogels in Biology and Medicine: From Molecular Principles to Bionanotechnology," Adv Mater 18, 1345-1360 (2006).

[3] F. Yu, X. Cao, Y. Li, L. Zeng, B. Yuan, and X. Chen, "An injectable hyaluronic acid/PEG hydrogel for cartilage tissue engineering formed by integrating enzymatic crosslinking and Diels-Alder 'click chemistry," Polym. Chem. 5, 1082-1090, 2014.

[4] P. Gupta P, K. Vermani, and S. Garg, "Hydrogels: from controlled release to pH-responsive drug delivery," Drug Discovery Today, 7, 569-579, 2002.

[5] P. C. Nicolson, "Continuous Wear Contact Lens Surface Chemistry and Wearability," Eye \& Contact Lens. 29, S30$2,2003$.

[6] J. Pozuelo, V. Compan, J. M. Gonzalez-Meijome, M. Gonzalez, and S. Molla, "Oxygen and ionic transport in hydrogel and silicone-hydrogel contact lens materials: An experimental and theoretical study," J Memb Sci., 452, 6272, 2014.

[7] Roach P, Farrar D, Perry CC. "Interpretation of protein adsorption: surface-induced conformational changes," $J$ Am Chem Soc., 127, 8168-8173, 2005.

[8] W. Norde, "Energy and Entropy Of Protein Adsorption," J Dispers Sci Technol, 13, 363-377, 1992.

[9] D. Luensmann and L. Jones, "Protein deposition on contact lenses: the past, the present, and the future," Contact Lens \& Anterior Eye, 35, 53-64, 2012.

[10] H. Thissen, Gengenbach T, du Toit R, Sweeney DF, Kingshott P, Griesser HJ, et al., "Clinical observations of biofouling on PEO coated silicone hydrogel contact lenses", Biomaterials, 31, 5510-5519, 2010.

[11] Banerjee, R. C. Pangule, and R. S. Kane, "Antifouling coatings: recent developments in the design of surfaces that prevent fouling by proteins, bacteria, and marine organisms," Adv Mater, 23, 690-718, 2011.

[12] X. Liu, Y. Xu, Z. Wu, and H. Chen, "Poly(N-vinylpyrrolidone)-modified surfaces for biomedical applications," Macromol Biosci, 13, 147-154, 2013.

[13] J. Wu, C. Zhao, R. Hu, W. Lin, et al., "Probing the weak interaction of proteins with neutral and zwitterionic antifouling polymers," Acta Biomater, 10, 751-760, 2014.

[14] Z. Cao, L. Mi, J. Mendiola, J. R. Ella-Menye, et al., "Reversibly switching the function of a surface between attacking and defending against bacteria," Angew Chem Int Ed Engl., 51, 2602-2605, 2012.

[15] Y. A. Krutyakov, A. A. Kudrinskiy, A. Y. Olenin, and G. V. Lisichkin, "Synthesis and properties of silver nanoparticles: advances and prospects", Russ Chem Rev., 77, 233-257, 2008.

[16] Yu H, Xu X, Chen X, Lu T, Zhang P, Jing X, "Preparation and antibacterial effects of PVA-PVP hydrogels containing silver Nanoparticles", J Appl Polym Sci., 103, 125-133, 2007.

[17] M.E. Nash, W. M. Carroll, P. J. Foley, G. Maguire, et al., "Ultra-thin spin coated crosslinkable hydrogels for use in cell sheet recovery-synthesis, characterisation to application," Soft Matter, 8, 3889-3899, 2012.

[18] J. Ma, Y. Sahai, “A Direct Borohydride Fuel Cell with Thin Film Anode and Polymer Hydrogel Membrane,” ECS Electrochem Lett., 1, F41-F43, 2012.

[19] F. Stokker-Cheregi, A. Matei, M. Dinescu, and C. E. Secu, "Photoluminescence of Eu-doped LiYF 4 thin films grown by pulsed laser deposition and matrix-assisted pulsed laser evaporation," J Phys D Appl Phys., 47, 045304-9, 2014.

[20] R. Cristescu, D. Mihaiescu, G. Socol, I. Stamatin, I. N. Mihailescu, and D. B. Chrisey, "Deposition of biopolymer thin films by matrix assisted pulsed laser evaporation," Appl Phys A., 79, 1023-1026, 2004. 